\title{
Tourism Domain Ontology Construction Method Based on Fuzzy Formal Concept Analysis
}

\author{
Yan Liu ${ }^{1}$,ShengQuan $\mathrm{Liu}^{2}$,Peng $\mathrm{Li}^{1}$ \\ 1.College of Information Science \& Engineering \\ Xinjiang University, \\ Urumqi, 830046, China \\ e-mail: liuyuxiu@xju.edu.cn \\ 2.Center of Modern Educational Technology \\ Xinjiang University \\ Urumqi, 830046, China \\ e-mail: liu@xju.edu.cn
}

\begin{abstract}
In this paper, fuzzy formal concept analysis is introduced to the tourism domain ontology construction process, first fuzzy formal concept analysis of uncertain information in the domain of tourism,then through the conceptual clustering generated fuzzy concept hierarchy, lastly mapping to get fuzzy ontology prototype. A example shows that the method is feasible and effective.
\end{abstract}

Keywords-Fuzzy Formal Concept Analysis; Tourism Domain; Domain Ontology

\section{INTRODUCTION}

Domain ontology [1] a shared, conceptualization, formal representation of knowledge system of the domain, using the class represents a concept, and support of Taxonomic and non-Taxonomic relations between classes. But in real life, people contact information is often uncertain, for example, in the domain of tourism, there is a lot of uncertain information, such as the satisfaction level of the tourists on scenic or scenic emphasis on the human and natural etc, but by the classical ontology supports formal concepts can not express uncertain information. Therefore, it is necessary to construct the Fuzzy Domain Ontology on a particular domain of uncertain information description.

The fuzzy ontology construction has already made some achievements. Xinxiang Tang[2] proposed a using MDA technology to develop the method of fuzzy ontology, using the MOF defines a fuzzy ontology modeling language FOML, and extended OWL into fuzzy OWL, according to fuzzy OWL and FOML mapping, using visual FOML development fuzzy ontology to improve the efficiency of fuzzy ontology. Yanhui Lv[3] introduced one kind based on the fuzzy UML class diagram of fuzzy ontology construction method, from the logic foundation of fuzzy UML class diagram illustrates the use of fuzzy ontology generation feasibility, and describes the fuzzy UML class diagram to fuzzy OWL DL ontology mapping method, gives the mapping rules and axioms of the creation process. Jun Zhai[4] based on linguistic variables of the fuzzy ontology model to do the research, by introducing the concept of fuzzy ontology of linguistic variables, using the RDF representation of fuzzy ontology, ontology and fuzzy concept are expressed as "resources". Aina He[5] put the Fuzzy Resource Description Framework(FRDF) as the base, introducing it into the ontology building tool protégé, carried it corresponding expand and modify to accommodate the establishment of fuzzy ontology.

This paper will combined Formal Concept Analysis(FCA) with Fuzzy Set Theory, putted forward a Fuzzy Formal Concept Analysis (FFCA) method and introduced to the tourism domain ontology construction process, by using the membership function of fuzzy set to represents the mapping relation of the objects and the attributes of a concept hierarchy, constructing the fuzzy tourism domain ontology, and gives an example verification. Compared to other fuzzy ontology construction method, the domain ontology's class constructed by this method can express the fuzzy information in the domain ontology, and can automatically generate domain ontology's concept hierarchy.

\section{FUZZY TOURISM DOMAIN ONTOLOGY CONSTRUCTION FRAMEWORK}

Apply the Fuzzy Formal Concept Analysis constructing fuzzy tourism domain ontology, the main idea is: used the fuzzy formal context to express the uncertain information obtained from the tourism domain information, then used the incremental algorithm constructed the fuzzy concept lattice in fuzzy formal context, and then using fuzzy concept clustering algorithm clustering fuzzy concept lattice automatically generated fuzzy concept hierarchy, Finally through mapping to get fuzzy ontology prototype. The construction process is shown in Figure 1 .

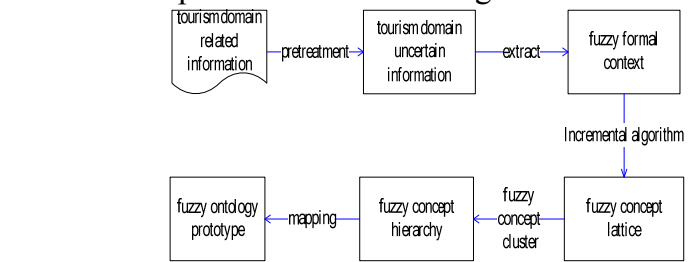

Figure 1. Tourism Domain Fuzzy Ontology Construction Method Flow Chart 


\section{TOURISM DOMAIN FUZZY FORMAL CONCEPT ANALYSIS}

\section{A. Related Concept}

To deal with the real world's specific domain of uncertain information, combine the Fuzzy Set Theory and Formal Concept Analysis (FCA), from specific areas of uncertainty information structure Fuzzy Form Background, then in the Fuzzy Form Background on the use of certain construction algorithm construct fuzzy concept lattice, this process is called Fuzzy Formal Concept Analysis.[6]

Definition 1. Fuzzy Formal Context. A fuzzy form background is expressed as $\mathrm{F}=(\mathrm{O}, \mathrm{A}, \mathrm{I})$, which $\mathrm{O}$ for a set of objects, $\mathrm{A}$ is an attribute set, mapping $\mathrm{I}$ is called membership function. This function satisfies:

$\mathrm{I}(\mathrm{o}, \mathrm{a})=\mu$, 其中 $\mathrm{o} \in \mathrm{O}, \mathrm{a} \in \mathrm{A}, \mu \in[0,1]$

Definition 2. Fuzzy Concept. Given a fuzzy formal context $\mathrm{F}=(\mathrm{O}, \mathrm{A}, \mathrm{I})$ and the threshold $\Phi_{d}$

$$
\begin{aligned}
& \text { for } \forall O_{1} \subseteq O, f\left(O_{1}\right)=\left\{a \in A \mid \forall o \in O_{1}, I(o, a) \geq \Phi_{d}\right\} \\
& \text { for } \forall A \subseteq A, g(A)=\left\{o \in O \mid \forall a \in A, I(o, a) \geq \Phi_{d}\right\}
\end{aligned}
$$

then fuzzy concept is a binary $C_{1}=\left(O_{1}, A\right), O_{1} \subseteq O, A \subseteq A, f\left(O_{1}\right)=A, g(A)=O_{1}$. Where $O_{1}$ and $\mathrm{A}_{1}$ were the extent and intent of the fuzzy concept $\mathrm{C}_{1}$.

Definition 3. Assumptions $\left(\mathrm{O}_{1}, \mathrm{~A}_{1}\right),\left(\mathrm{O}_{2}, \mathrm{~A}_{2}\right)$ are two fuzzy concept of fuzzy formal context $(\mathrm{O}, \mathrm{A}, \mathrm{I})$, If and only if $O_{1} \subseteq O_{2}$, then $A_{1} \subseteq A_{2}$, then $\left(\mathrm{O}_{1}, \mathrm{~A}_{1}\right)$ is the sub-concept of $\left(\mathrm{O}_{2}, \mathrm{~A}_{2}\right)$, and $\left(\mathrm{O}_{2}, \mathrm{~A}_{2}\right)$ is the super-concept of $\left(\mathrm{O}_{1}, \mathrm{~A}_{1}\right)$.

Table I is the example of Scenic uncertain information in the tourism domain. The object is 5 scenic instances, near or far from the urban areas, the level of scenic ticket price, the level of visitor satisfactions, scenic emphasis on the human and natural is the attributes. Under the guidance of domain experts, extracting fuzzy formal context and standardization(set up the threshold $\Phi_{d}=0.4$, membership degree greater than the threshold for original, joining the concept generation, otherwise set up 0). Here are a relevant tourism domain scenic fuzzy formal context.

TABLE I. FUZZY FORMAL CONTEXT

\begin{tabular}{|c|c|c|c|c|c|c|c|c|}
\hline & \multicolumn{2}{|c|}{$\begin{array}{c}\text { away from } \\
\text { the urban }\end{array}$} & \multicolumn{2}{c|}{ ticket price } & \multicolumn{2}{c|}{$\begin{array}{c}\text { visitor } \\
\text { satisfactions }\end{array}$} & \multicolumn{2}{c|}{ the emphasis } \\
\hline $\mathrm{o}_{\mathrm{i}}$ & Far a & Near b & High c & Low d & High $\mathrm{e}$ & Low $\mathrm{f}$ & Natural g & Human h \\
\hline 1 & 0.8 & 0 & 0 & 0.7 & 0 & 0.6 & 0.9 & 0 \\
\hline 2 & 0.9 & 0 & 0 & 0.8 & 0 & 0.8 & 0.8 & 0 \\
\hline 3 & 0.7 & 0 & 0.8 & 0 & 0.7 & 0 & 0 & 0.8 \\
\hline 4 & 0 & 0.6 & 0.7 & 0 & 0.7 & 0 & 0 & 0.8 \\
\hline 5 & 0 & 0.8 & 0.9 & 0 & 0.8 & 0 & 0 & 0.6 \\
\hline
\end{tabular}

\section{B. Construction of Tourism Domain Fuzzy Concept} Lattice

Traditional generating concept lattice has two main ways: batch method, incremental method. Incremental method proved to be excellent[6], so use incremental method constructing the fuzzy concept lattice.

Definition 4. Fuzzy Concept Lattice. Denoted CS(F) as all the fuzzy concept of fuzzy formal context $\mathrm{F}$, the structure of CS(F) is a partial ordering relation. If satisfied
$O_{1} \subseteq O_{2}$, then $\left(O_{1}, A_{1}\right) \leq\left(O_{2}, A_{2}\right)$, through this relationship get ordered set $\underline{C S(F)}=(C S(F), \leq)$ called fuzzy concept lattice of the fuzzy form background $F$.

Algorithm 1. Fuzzy Concept Lattice Incremental Construction Algorithm

Use Intent $(\mathrm{C})$ represents the intent of the concept node $\mathrm{C}$, Extent $(\mathrm{C})$ represents the extent of the concept node $\mathrm{C}$.

Step 1: Fuzzy concept lattices $\mathrm{CS}(\mathrm{F})$ is initialized to empty;

Step 2: Remove an object $\mathrm{x}$ from fuzzy formal context $\mathrm{F}$, Form a node $(\{\mathrm{x}\}, \mathrm{f}(\{\mathrm{x}\}))$, if there is no node $\mathrm{C}$ in $\mathrm{CS}(\mathrm{F})$ makes, then put the node $(\{\mathrm{x}\}, \mathrm{f}(\{\mathrm{x}\}))$ into $\mathrm{CS}(\mathrm{F})$;

Step 3: Scanning all the nodes in $\operatorname{CS}(\mathrm{F})$, find out all the intent is less than or equal to the new object $\mathrm{x}$ intent node $\mathrm{C}($ Intent $(\mathrm{C}) \subseteq \mathrm{f}(\{\mathrm{x}\}))$, then the node $\mathrm{C}$ is update node. Updated wihe each update node $(\operatorname{Extent}(C) \cup\{x\}$, Intent $(C))$, edges do not update. Turn Step5;

Step 4: Scanning all the nodes in CS(F). If the node C and $\mathrm{x}$ intent intersection is not equal to any node in the intent of CS(F), the defined as the generation sub-node(the same intent node take the biggest extent), each generation sub-node and the $\mathrm{x}$ together generate a new generation node $(\operatorname{Extent}(\mathrm{C}) \cup\{\mathrm{x}\}$, Intent $(\mathrm{C}) \cap \mathrm{f}(\mathrm{x}))$. If there is update node of the new generation node in $\mathrm{CS}(\mathrm{F})$, then update these node, else add the new generation node in $\mathrm{CS}(\mathrm{F})$, and connecting new generation node to its sub-node and super-node;

Step 5: Until all of the objects are added in the $\mathrm{CS}(\mathrm{F})$, else turn Step 2;

Step 6: Search for all the nodes without child nodes in $\mathrm{CS}(\mathrm{F})$, if such a node with more than one, then generate a bottom node( $\varnothing, \mathrm{A})$, increase the edges from the bottom node to such node. Search all the node without a parent node, if such a node with more than one, then generate the top node $(\varnothing, \mathrm{A})$, joined $\mathrm{CS}(\mathrm{F})$, and increased the edges from the top node to such node.

Figure 2 shows the fuzzy concept lattice which based on the use of Algorithms 1 by the fuzzy formal context of table I .

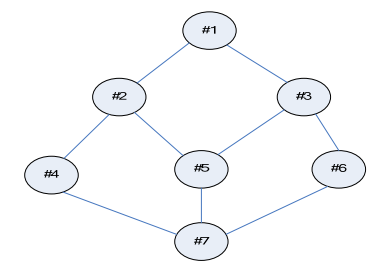

Figure 2. Fuzzy Concept Lattice

Table II shows the constitute and the fuzzy parameters value of the fuzzy formal concept, for convenience of description, the eight attributes of the fuzzy formal concept in table I are represented by symbols a, b, c, d, e, f, g, h to replace.

\begin{tabular}{|l|l|}
\multicolumn{1}{c}{ TABLE II. } & FUZZY FORMALCONCEPTCONSTITUTION TABLE \\
\hline $\begin{array}{l}\text { Node } \\
\text { number }\end{array}$ & FuZZy formal concept constitute \\
\hline
\end{tabular}




\begin{tabular}{|c|c|}
\hline 1 & $(\{1,2,3,4,5\},\{\varnothing\})$ \\
\hline 2 & $(\{1,2,3\},\{a(0.7)\})$ \\
\hline 3 & $(\{3,4,5\},\{\mathrm{c}(0.7), \mathrm{e}(0.7), \mathrm{h}(0.6)\})$ \\
\hline 4 & $(\{1,2\},\{\mathrm{a}(0.8), \mathrm{d}(0.7), \mathrm{f}(0.6), \mathrm{g}(0.8)\})$ \\
\hline 5 & $(\{3\},\{\mathrm{a}(0.7), \mathrm{c}(0.7), \mathrm{e}(0.7), \mathrm{h}(0.8)\})$ \\
\hline 6 & $(\{4,5\},\{\mathrm{b}(0.6), \mathrm{c}(0.7), \mathrm{e}(0.7), \mathrm{h}(0.6)\})$ \\
\hline 7 & $(\{\varnothing\},\{\mathrm{a}(1), \mathrm{b}(1), \mathrm{e}(1), \mathrm{d}(1), \mathrm{e}(1), \mathrm{f}(1), \mathrm{g}(1), \mathrm{h}(1)\})$ \\
\hline
\end{tabular}

\section{A. ClusteringGenerated FuzzyConcept Hierarchy}

In the traditional concept lattice, formal concept is to use mathematical method to produce, the objects with the very small difference of the relevant attribute values can be classified into a formal concept, at higher levels can be clustered into a concept. Based on the method, we can use fuzzy conceptual clustering technique to cluster the concepts together into a number of clustering, use similarity threshold Ts to determine whether the two concepts clustering. The concept of clustering can have the following properties[7]:

(1) Fuzzy conceptual clustering has hierarchical relationships, its hierarchical relationships can derived from the fuzzy formal concept of fuzzy formal concept lattice. That is if the two clustering in the form concept have a super-sub relationship, the two clustering also have a super-sub relationship.

(2) A fuzzy formal concept must belong to at least one fuzzy conceptual clustering, but can belong to more than one fuzzy conceptual clustering.

Definition 5. The concept similarity of the fuzzy formal concept $(\mathrm{O} 2, \mathrm{~A} 2)$ and its sub-concepts $(\mathrm{O} 1, \mathrm{~A} 1)$ can be defined as:

$$
\begin{aligned}
& \operatorname{Sim}\left(C_{1}, C_{2}\right)=\frac{\varphi\left(O_{1}\right)}{\varphi\left(O_{2}\right)} \\
& \varphi\left(O_{i}\right)=\sum_{o \in O_{i}} \mu\left(o, A_{i}\right)
\end{aligned}
$$

\section{Algorithm 2. Based on Concept Similarity Concept}

\section{Clustering Algorithm}

Input: Fuzzy concept lattice CS(F), similarity threshold

Ts Output: Fuzzy concept clustering CS'(F)

Step 1: Pretreatment, read the edge set CXCY of $\mathrm{CS}(\mathrm{F}),(\mathrm{CX}$ is the parent nodes of $\mathrm{CY}$, edges including the super-node and the sub-node and the node's similarity parameters):

Step 2: Sequential search each edge $\mathrm{CiCj}$ in the edge set $(\mathrm{CiCj} \in \mathrm{CXCY}), \quad$ Computing concept similarity $\operatorname{Sim}(\mathrm{Ci}, \mathrm{Cj})$;

Step 3: if $\mathrm{Sim}(\mathrm{Ci}, \mathrm{Cj})>\mathrm{Ts}$, clustering $\mathrm{Ci}, \mathrm{Cj}$ to generate a new node Cij; Otherwise turn Step 5;

Step 4: Update the node $(\{\mathrm{OX} \cup \mathrm{OY}\},\{\mathrm{AX} \cup \mathrm{AY}\})$, and to replace super-node $\mathrm{Ci}$; Update the edge set, the super-node of $\mathrm{Ci}$ connected to the $\mathrm{Cij}$, $\mathrm{Cij}$ connected to the sub-node of $\mathrm{Cj}$, delete $\mathrm{Cj}$;

Step 5: turn to Step 2, Until there is no edge CiCj make such that $\mathrm{Sim}(\mathrm{CiCj})>\mathrm{Ts}$ was established;

Step 6: edge set store into CS'(F).

Compute the concept similarity Sim of fuzzy formal concept which have the super-sub relationship in the fuzzy concept lattice. The results are as follows:

$\operatorname{Sim}(\# 4, \# 2)=4.14$,

$\operatorname{Sim}(\# 5, \# 3)=1.45, \quad \operatorname{Sim}(\# 6, \# 2)=1.3$

Take the similarity threshold $\mathrm{Ts}=3$, using the Algorithm 2 to clustering the fuzzy concept lattice which is shown in Figure 2. After clustering, the fuzzy concept hierarchy was shown as Figure 3. Due to node 1 and node 7 respectively is one of the largest concept and empty concept in the domain, so in the fuzzy ontology construction not consideration, cut off its relationship with other node.

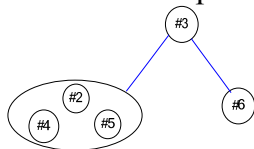

Figure 3. Fuzzy Concept Hierarchy

\section{B. Mapping Get Fuzzy Ontology Prototype}

According to fuzzy concept hierarchy and fuzzy ontology elements in the corresponding relationship, using the mapping rules can constructed the fuzzy ontology prototype. Mapping rules as shown in Figure 4.

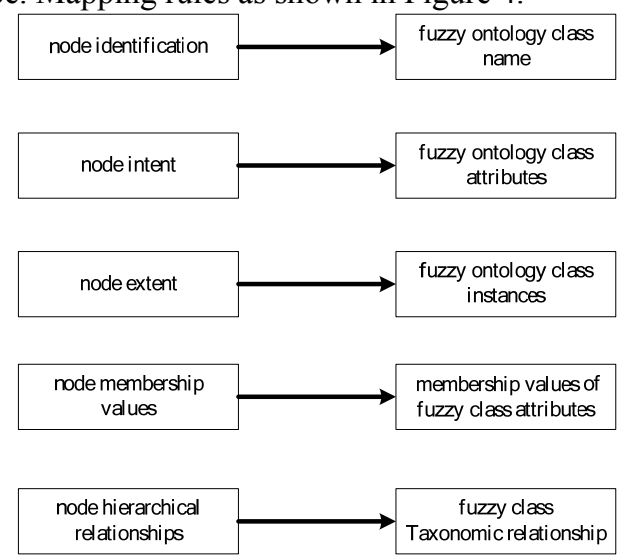

Figure 4. Fuzzy Concept Hierarchy and Fuzzy Ontology Mapping Rules

The fuzzy concept hierarchy(shown as Fig. 3) using the mapping rules can get the fuzzy ontology(shown as Figure 5), Three node after clustering $\{\# 3\}$ 、 $\{\# 2 、 \# 4 、 \# 5\},\{\# 6\}$ represent a fuzzy class of the travel ontology, named as fuzzy class A,B,C.

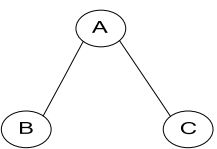

Figure 5. Fuzzy Ontology Prototype

In summary, this paper starting from the fuzzy formal context of a given tourism domain, first constructs a fuzzy concept lattices, then clustering generated the fuzzy concept hierarchy, finally mapping to get a fuzzy travel domain 
ontology prototype(shown as Figure 5). We can see, the fuzzy tourism domain ontology class not only can express the fuzzy information in the domain ontology, but also can automatically generate domain ontology's concept hierarchy. For example, In the fuzzy class A, its attributes are ticket price High(0.7), visitor satisfactions High(0.7), the emphasis $\operatorname{Human}(0.6)\}$, its Instances are the Scenic $\{3,4,5\}$, it is the super-class of fuzzy class B and fuzzy class C. In the fuzzy class B, its attributes are \{ away from the urban $\operatorname{Far}(0.7)$, ticket price $\operatorname{High}(0.7)$, ticket price $\operatorname{Low}(0.7)$, visitor satisfactions Low(0.6), the emphasis Natural(0.8), the emphasis Human $(0.8)\}$, its Instances are the Scenic $\{1,2,3\}$, it is the sub-class of fuzzy class A. In the fuzzy class C, its attributes are \{ away from the urban $\operatorname{Far}(0.6)$, ticket price $\operatorname{High}(0.7)$, visitor satisfactions $\operatorname{High}(0.7)$, the emphasis Human $(0.6)\}$, its Instances are the Scenic $\{4,3\}$, it is the sub-class of fuzzy class A.

Through the mapping rules get the fuzzy ontology prototype, also need the participation of domain experts, add non-Taxonomic relations between the fuzzy class and properties, axioms, instances, expansion the fuzzy ontology prototype, to get More perfect fuzzy domain ontology.

\section{CONCLUSION}

This paper proposes a fuzzy formal concept analysis applied to tourism domain, constructing and generate the fuzzy tourism domain ontology method. Formal analysis in the expression of fuzzy uncertainty information has a unique advantage, it constructs fuzzy concept lattices to deal with uncertain information, fuzzy concept lattice clustering as the domain ontology prototype construction way, and can automatically generate domain ontology's concept hierarchy. The example shows the feasibility and effectiveness of this method. The next work is to automatically construct fuzzy formal context and different fuzzy conceptual clustering algorithm research.

\section{ACKNOWLEDGMENT}

The Universities and colleges jointly funded project of Xinjiang University under Grant No.XY110121

$$
\text { REFERENCES }
$$

[1]. Borst W N.Construction of Engineering Ontologies for Knowledge Sharing and Reuse. C.Enschede: University of Twente,(1997)

[2]. Xinxiang Tang, Huaikou Liao. Generationof $\mathrm{MDA}_{\ulcorner}$BasedFuzzyOntology. J. Journal of Applied Sciences. 25,541-543(2007)

[3]. Yanhui Lv, Zongmin Ma, Fu Zhang. An Approach to Fuzzy Ontology Framing Based on Fuzzy Conceptual Model. J. Journal of Northeastern University(Natural Science).30,1262-1265(2009)

[4]. Jun Zhai, Jianfeng Li, Yan Chen. Representation and Construction for Fuzzy Ontology of Linguistic Variables. J.Computer \& Digital Engineering.9,113--114(2009)

[5]. Junmin Luo, Yuyun Wu, Bin Wu. Research on the Fuzzy Ontology and it's Evolution. J. Microelectronics \& Computer.5,140-143(2011)

[6]. Zongtian Liu, Yu Qiang, Wen Zhou, et al. A Fuzzy Concept Lattice Model and Its Incremental Construction Algorithm. J. Chinese Journal of Computers.2,184-187(2007)
[7]. Q.T. Tho,S.C. Hui,A.C.M. Fong and T.H. Cao,Automatic Fuzzy Ontology Generation for Semantic Web[J], IEEE Trans. Knowledge and Data Eng.,vol. 18, no. 6, pp. 842-856, (2006) 